\title{
Altered Insula Response to Taste Stimuli in Individuals Recovered from Restricting-Type Anorexia Nervosa
}

\author{
Angela Wagner ${ }^{1,2}$, Howard Aizenstein', Laura Mazurkewicz', Julie Fudge ${ }^{3}$, Guido K Frank ${ }^{1,4}$, \\ Karen Putnam ${ }^{5}$, Ursula F Bailer ${ }^{1,6}$, Lorie Fischer' and Walter H Kaye*, \\ 'Department of Psychiatry, School of Medicine, Western Psychiatric Institute and Clinic, University of Pittsburgh, Pittsburgh, PA, USA; \\ ${ }^{2}$ Department of Child and Adolescent Psychiatry, J.W.-Goethe University of Frankfurt, Frankfurt, Germany; ${ }^{3}$ Departments of Psychiatry, and \\ Neurobiology and Anatomy, University of Rochester Medical Center, Rochester, NY, USA; ${ }^{4}$ Department of Psychiatry, University of California, \\ San Diego, La Jolla, CA, USA; ${ }^{5}$ Department of Environmental Health, Division of Epidemiology and Biostatistics, School of Medicine, University \\ of Cincinnati, Cincinnati, OH, USA; ${ }^{\circ}$ Department of General Psychiatry, Medical University of Vienna, University Hospital of Psychiatry, \\ Vienna, Austria
}

\begin{abstract}
Anorexia nervosa (AN) is an illness characterized by aversion to ingestion of normally palatable foods. We examined whether there is a primary disturbance of taste processing and experience of pleasure using a sucrose/water task in conjunction with functional magnetic resonance imaging (fMRI). To avoid confounding effects of illness, 16 women recovered from restricting-type AN were compared to 16 control women $(\mathrm{CW})$. We used a region of interest-based $\mathrm{AMRI}$ approach to test the idea that individuals with AN have differential neural activation in primary and secondary taste cortical regions after sucrose and water administration. Compared to CW, individuals recovered from AN showed a significantly lower neural activation of the insula, including the primary cortical taste region, and ventral and dorsal striatum to both sucrose and water. In addition, insular neural activity correlated with pleasantness ratings for sucrose in CW, but not in AN subjects. Altered taste processing may occur in AN, based on differences in activity in insular-striatal circuits. These data provide the first evidence that individuals with AN process taste stimuli differently than controls, based on differences in neural activation patterns.
\end{abstract}

Neuropsychopharmacology (2008) 33, 513-523; doi: I0.1038/sj.npp. I 301443; published online 9 May 2007

Keywords: taste; anorexia nervosa; insula; striatum; sucrose; pleasantness

\section{INTRODUCTION}

Anorexia nervosa (AN) is a disorder of unknown etiology characterized by restricted eating, a relentless pursuit of thinness, and obsessive fears of becoming fat. The onset of AN tends to occur in early adolescence with approximately $90 \%$ of subjects being female (American Psychiatric Association, 1994). AN is often characterized by a chronic and relapsing course (Herzog et al, 1996) and it has the highest death rate of any psychiatric disorder (Sullivan, 1995). Moreover, the hospital utilization rate for AN is higher than for any other psychiatric disorder, aside from schizophrenia (McKenzie and Joyce, 1992). In part, chronicity and the frequent need for hospitalization are a consequence of the limited efficacy of current pharmacologic and psychological treatments. While several lines of

\footnotetext{
*Correspondence: Dr WH Kaye, Department of Psychiatry, School of Medicine, Western Psychiatric Institute and Clinic, University of Pittsburgh, Iroquois Building, Suite 600, 38 I I O'Hara Street, Pittsburgh, PA 152 13, USA, Tel: + | 412647 9845, Fax: + | 4126479740 ,

E-mail: kayewh@upmc.edu

Received 28 November 2006; revised 13 March 2007; accepted 29 March 2007
}

evidence implicate genetically mediated neurobiological factors as contributing to the development of AN (Bailer and Kaye, 2003; Bulik et al, 2006), the lack of understanding of the pathophysiology of this illness has hindered the development of more effective treatments. For example, AN is characterized (APA, 2000) as an eating disorder (ED). Despite this categorization, it remains unknown whether individuals with AN have a primary disturbance of pathways that modulate feeding, or whether disturbed appetite is secondary to other phenomena, such as anxiety or obsessional preoccupation with weight gain.

The regulation of appetite and feeding are complex phenomena, involving peripheral and central pathways. Until recently, few tools were available that could directly investigate the brain in vivo in humans. Brain imaging studies performed in the past decade in human and nonhuman primates show that a primary taste cortex resides in the rostral insula and adjoining frontal operculum (Scott et al, 1986; Yaxley et al, 1990; Faurion et al, 1999; Schoenfeld et al, 2004). Some studies argue that these regions provide a representation of food in the mouth that is independent of hunger, and thus of reward value (Rolls, 2005). The responsiveness of taste neurons in secondary 
regions, such as the orbitofrontal cortex (OFC), compute the hedonic value of food (O'Doherty et al, 2000; Kringelbach et al, 2003; Rolls, 2005). Other studies (Small et al, 2001) raise the possibility that the insula and OFC contribute to feeding behavior by encoding changes in the value of food reward in addition to sensory processing, suggesting overlapping representations of sensory and affective processing of taste.

Previous brain imaging studies have shown pictures of food to emaciated and malnourished AN individuals. These studies found altered activity not only in the insula and OFC, but in broad regions including mesial temporal, parietal, and the anterior cingulate cortex when ill $\mathrm{AN}$ subjects were compared to controls (Nozoe et al, 1993; Ellison et al, 1998; Naruo et al, 2000; Gordon et al, 2001; Uher et al, 2004). In addition, one study used pictures of food and functional magnetic resonance imaging (fMRI) to study individuals who were recovered from AN (Uher et al, 2003). That study found evidence of altered function in anterior supergenual cingulate cortex and medial prefrontal region. Together these studies support the possibility that cortical regions implicated in regulation of feeding behavior might be disturbed in AN.

When individuals with $\mathrm{AN}$ are underweight and malnourished, they display considerable disturbances of most physiological systems. Thus it is not possible to determine whether abnormal findings are cause or consequence of starvation. To avoid the possible confounding effect of starvation and emaciation on response to taste, we studied individuals who were remitted from restricting-type AN. Approximately $50 \%$ of individuals who have AN recover, in the sense that their weight and nutritional status normalize (Couturier and Lock, 2006). However, these individuals often continue to have persistent dysphoric mood, obsessionality, and body image concerns, which are modest compared to the underweight state (Wagner et al, 2006a). Since such symptoms are present in childhood, before the onset of $\mathrm{AN}$, they may reflect traits that contribute to vulnerabilities to develop AN.

The purpose of this study was to determine whether individuals recovered from restricting-type $\mathrm{AN}$ have an abnormal physiological response to sugar, a pleasant taste, or water, a neutral contrast taste, in the primary or in secondary taste-modulation cortical regions. Individuals recovered from AN were compared to matched control women $(\mathrm{CW})$, and sugar and water were administered in a blind, controlled manner. We used a $10 \%$ concentration of sucrose. This concentration is present in soft drinks and is a level of sweetness preferred by the general public (Drewnowski et al, 1987; Sunday and Halmi, 1990). In primates and healthy humans, both sugar (Rolls, 2005) and water (Scott et al, 1986; Yaxley et al, 1990; Zald and Pardo, 2000; De Araujo et al, 2003a) ingestion activates neurons in the primary and secondary cortical taste areas. We hypothesize that AN women might have a disturbance of these higher order pathways modulating the sensory and hedonic aspects of feeding. Individuals with AN do not simply have a loss of appetite. Rather, they display puzzling symptoms that include the avoidance and fear of normally pleasurable foods. Yet, they are also obsessed with such foods, and often 'window-shop' these foods in supermarkets, collect recipes and cookbooks, and cook such foods for others.
The anterior insula, and many of these secondary tastemodulatory regions, also contribute to emotional regulation (Fudge et al, 2005; Paulus and Stein, 2006; Stein et al, 2006). It is clinically well recognized that anxiety often accompanies eating in individuals with AN. Thus we compared sugar to water, and asked subjects to rate their anxiety and the pleasantness of the taste stimuli. We wanted to determine whether the signal in response to sugar was markedly different than the signal in response to water, and if the difference was related to anxiety.

\section{MATERIALS AND METHODS}

\section{Sample Collection}

Sixteen women who had recovered from restricting-type AN were recruited. Subjects were previously treated in the EDs treatment program at the Western Psychiatric Institute and Clinic (Pittsburgh, PA) or were recruited through advertisements. To be considered 'recovered', subjects had to (1) maintain a weight above $90 \%$ average body weight (Metropolitan Life Insurance Company, 1959), (2) have regular menstrual cycles; and (3) have not binged, purged, or engaged in significant restrictive eating patterns for at least 1 year before the study. Restrictive eating pattern was defined as regularly occurring behaviors, such as restricting food intake, restricting high-caloric food, counting calories, and dieting. Additionally, subjects must not have used psychoactive medication such as antidepressants or met criteria for alcohol or drug abuse or dependence, major depressive disorder (MDD), or severe anxiety disorder within 3 months of the study. Sixteen age-matched healthy $\mathrm{CW}$ were recruited through local advertisements. The CW had no history of an ED or any psychiatric, serious medical, or neurological illness. They had no first-degree relative with an ED. They had normal menstrual cycles and had been within normal weight range since menarche. CW were not on medication, including herbal supplements.

This study was conducted according to the institutional review board regulations of the University of Pittsburgh, and all subjects gave written informed consent. The MR imaging was performed during the first 10 days of the follicular phase for all subjects. The MR study was performed at 0900 hours. All subjects had the same standardized breakfast (bread, butter, marmalade, milk/ juice; no coffee) on the morning ( 0700 hours) of the study. This design was chosen to standardize the subjects' state of satiety.

\section{Assessments}

A comprehensive battery of clinical interviews and selfassessments was designed to assess symptoms typical in individuals with EDs. Trained doctoral level clinicians with experience in EDs administered the clinical interviews. All instruments have demonstrated good reliability and validity. A comprehensive description of the assessment is described elsewhere (Wagner et al, 2006a). After scanning, subjects were asked to provide subjective pleasantness ratings for each individual taste (using a scale from $+3=$ very pleasant, $0=$ neutral, through to $-3=$ very unpleasant). In addition, they were asked how anxious they 
got $+3=$ very anxious, $0=$ no effect, $-3=$ calm) for each individual taste.

\section{Experimental Paradigm}

The paradigm was based on a task developed in our group and described previously (Frank et al, 2003). In the present task, two different stimuli were presented: 10\% sucrose (Fisher Scientific, USA) and distilled water. These solutions are described in the literature as pleasant and neutral, respectively (Drewnowski et al, 1987). The paradigm consisted of six blocks of 20 trials each. Within blocks, stimuli were presented either sequentially or pseudorandomly. This paper presents data for the two pseudorandom blocks as the data for the sequential blocks are driven by habituation effects (data not shown). Thus, sucrose and water were presented randomly, but in the same 'random' order for all subjects (hence pseudorandom). Within these pseudorandom blocks, the number of sampling scans of sucrose and water was the same resulting in a total of 20 for both. Each taste stimulus consisted of $1 \mathrm{ml}$ volume of liquid and was delivered every $20 \mathrm{~s}$ through one of two tubes in the buccal region. Subjects were trained to perform one tongue motion (swishing the solutions across the tongue) after each application of taste stimulant, to wash the taste stimulus around the mouth and stimulate taste buds.

\section{Apparatus}

The macronutrient solutions were contained in two $25 \mathrm{ml}$ syringes, which were attached to a semiautomatic and programmable customized syringe pump (J-Kem Scientific, St Louis, MO), positioned in the scanner control room (Frank et al, 2003). Tastes were delivered to the subjects via two separate approximately 10-m long FDA approved food grade Teflon tubes (Cole-Parmer Vernon Hills, IL). The syringes were also attached to a computer-controlled valve system, which enabled the two solutions to be delivered independently along the tubing. Taste delivery was controlled by E-Prime (Psychology Software Tools, Inc., Pittsburgh, PA) software operating on a PC positioned in the control room. The stimuli were also synchronized with MR scanning.

\section{Scanning Procedures}

Imaging data were collected with a 3T Signa scanner (GE Medical Systems). High-resolution anatomical images $\left(1.5 \mathrm{~mm}^{3}\right)$ were acquired for each subject. Additionally, T1 structural images were acquired with a $3.2 \mathrm{~mm}$ thickness (in-plane with the functional images). These had 36 oblique axial slices oriented parallel to a line from the anterior to posterior commissures (the AC-PC line), with an in-plane resolution of $0.78 \mathrm{~mm}^{2}$ and with a field of view of $200 \mathrm{~mm}^{2}$. Functional images were acquired using a one-shot reverse spiral pulse sequence (Noll et al, 1995; Bornert et al, 2000) with $\mathrm{TE}=26 \mathrm{~ms}$ and $\mathrm{TR}=2000 \mathrm{~ms} ; 30$ oblique axial slices were acquired with an in-plane resolution of $64 \times 64$ with $3.125 \mathrm{~mm}^{2}$ pixels and a slice thickness of $3.2 \mathrm{~mm}$, with a field of view of $200 \mathrm{~mm}^{2}$. The presentation of stimuli was synchronized with the scanning such that 10 2-s images were acquired per trial.

\section{Image Analysis}

Data preprocessing. Motion correction was performed using a six-parameter linear algorithm (Woods et al, 1998). A linear detrending algorithm was also carried out, using only data within three standard deviations of the mean to estimate the linear trend. Global normalization was performed multiplicatively to give each subject a mean intensity of 3000 . All analyses were conducted on a singlesubject basis on non-cross-registered data.

ROI selection. We used an a priori region of interest (ROI) approach, with the regions defined anatomically in each individual's anatomic space. In order to characterize higher order cortical taste regions, we selected the insula, OFC (BA 11), amygdala, and anterior cingulate cortex. Our group recently reported that remitted $A N$ had evidence of increased Dopamine (DA) D2/D3 receptor binding in the anterior ventral striatal regions (Frank et al, 2005). DA has been reported to be released in response to taste of food in animals and humans (Mirenowicz and Schultz, 1994; Schultz, 1998; Small et al, 2003b). Thus it is possible that remitted $\mathrm{AN}$ have an altered DA response to feeding. In addition, our group has found that remitted $\mathrm{AN}$ have altered responses to monetary reward, compared to $\mathrm{CW}$, and fail to appropriately discriminate positive and negative feedback (Wagner et al, 2005). This latter study raised the possibility that remitted AN have dysregulated corticostriatal response to salient stimuli in general, and not just food. For these reasons, we included exploratory assessments of fMRI response in striatal regions where we previously found disturbances of D2/D3 binding (Frank et al, 2005). We also explored striatal regions known to receive strong inputs from the anterior insula, a circuit implicated in the modulation of feeding (Small et al, 2001; Kelley et al, 2002; Fudge et al, 2005).

Automated labeling pathway. An automated approach for defining the ROIs was used, as described in Wu et al (2006). The anatomical ROIs were chosen based on a literature review of previous studies. Bilateral BA11, insula, anterior cingulate cortex, and amygdala were defined in the Automatic Anatomical Labeling (aal) map obtained from the MRIcro software package (Tzourio-Mazoyer et al, 2002). The subdivisions for the striatum were chosen based on criteria defined previously by Drevets et al (2001). In order to define each ROI for each single subject in her own space, the following steps were performed. First, each subject's low-resolution anatomical image was cross-registered to her high-resolution anatomical image, using a six-parameter linear algorithm (Woods et al, 1998). Then the Montreal Neurological Institute single-subject high-resolution anatomical image (the Colin brain) was aligned with each subject's high-resolution anatomical image using a fully deformable demons-based algorithm implemented in the Insight Segmentation and Registration Toolkit (www. itk.org). Each ROI from the aal map was then put onto each subject's SPGR. Then a gray matter mask was applied to each label, using the FAST algorithm from the FSL package (Zhang et al, 2001). The labeling of each subject's ROIs was then visually inspected to assure accurate mappings. 
Time series analysis. For each subject and for each ROI, a mean time series was extracted. The time series for all voxels in each region was first calculated as the percent signal change across the 10 different scans of a trial, that is, the percent change of signal intensity in all voxels from the first scan of the trial. The mean signal for each trial-type and for each region was then calculated and again averaged. The resulting time series data (10 time points for each subject for each ROI; each time point is $1 \mathrm{~s}$ ) were then used in the statistical analyses described below.

Statistical analysis. Region-of-interest fMRI time series data were analyzed using JMP (SAS Institute, Inc.). Comparisons within and between groups were performed using analyses of variance method, with factors for group (patient $v s$ control), time point (ie scan numbers 2-10), and condition (sucrose $v s$ neutral).

Standard statistical software package (SPSS Version 12) was used for all analyses of behavioral and demographic data. Comparisons between the two groups were made using Wilcoxon rank-sum tests. The exact levels were used due to the small sample sizes. Pearson's correlation coefficients were also computed using the mean percentage signal change across all trials.

\section{RESULTS}

\section{Behavioral Results}

Women recovered from $\mathrm{AN}$ and $\mathrm{CW}$ were of similar age (Table 1). The remitted AN subjects had a significantly lower current BMI than the CW, although mean BMI for both were within a normal range. As expected, at the lowest point, lifetime BMI was significantly less in AN compared to CW. AN had the onset of their ED when they were $16.4 \pm 3.1$ years old, and had been recovered for $45.7 \pm 30.2$ months.

Ten subjects in the AN group had a lifetime history of MDD and eight subjects had a lifetime history of obsessive- compulsive disorder. Additional lifetime comorbidity included alcohol abuse/dependence (two subjects), and drug abuse/dependence (one subject). None of the AN subjects had a history of any psychotic disorder. AN subjects had significantly higher values for trait and state anxiety (Spielberger Stait-Trait Anxiety Inventory (Spielberger et al, 1970)), depression (Beck Depression Inventory (Beck et al, 1961) BDI), and harm avoidance (Cloninger et al, 1994) in comparison to CW (Table 1).

\section{FMRI Results}

The three-way interaction for all regions was not significant (data not shown), indicating that the groups across conditions and across time behaved similarly. A two-way comparison of condition $\times$ time showed no significant differences across the regions, suggesting that the total group behaved similarly for sugar and water. Another two-way comparison was carried out, in which we tested differences between the groups to the taste stimuli in general, independent of whether the taste was sugar or water. This analysis, collapsed across conditions, revealed ROIs where the shape of the BOLD response differed over time between the groups (Table 2). The AN showed reduced response over time, regardless of condition (eg sucrose or water) for the insula bilaterally (Figure 1). In addition, broad regions of the striatum including the dorsal and middle caudate, and dorsal and ventral putamen (Figure 2) as well as the anterior cingulate cortex showed similar reduced responses compared to controls. In contrast, $\mathrm{AN}$ and $\mathrm{CW}$ showed a similar response to sugar and water in the anterioventral striatum (AVS), amygdala, and OFC (BA 11).

For CW, the signal activity (mean percentage signal change) of the insula (Figure 3 ) was significantly positively related to ratings for the pleasantness of the sucrose taste (left: $r=0.648, P=0.007$; right: $r=0.608, P=0.01$ ). Similarly, CW showed significant positive relationships between

Table I Group Comparisons of Demographic Variables and Assessment Data

\begin{tabular}{|c|c|c|c|c|c|}
\hline & \multicolumn{2}{|c|}{$C W(n=16)$} & \multicolumn{2}{|c|}{ AN $(n=16)$} & \multirow[b]{2}{*}{ Exact significance } \\
\hline & Mean & SD & Mean & SD & \\
\hline Current BMI $\left(\mathrm{kg} / \mathrm{m}^{2}\right)$ & 22.9 & 2.2 & 20.7 & 2.2 & 0.005 \\
\hline Lowest past BMI $\left(\mathrm{kg} / \mathrm{m}^{2}\right)$ & 20.6 & 1.6 & 14.1 & 2.1 & $<0.001$ \\
\hline Length of recovery (months) & & & 45.7 & 30.2 & \\
\hline BDI (Total) & 1.8 & 2.1 & 6.6 & 6.6 & 0.021 \\
\hline Harm avoidance $(\mathrm{TCl})$ & 9.9 & 5.6 & 17.5 & 6.5 & 0.003 \\
\hline Spielberger Trait Anxiety & 27.6 & 4.1 & 41.3 & 11.7 & $<0.001$ \\
\hline Anxiety for sucrose (scan) & 0.3 & 1.2 & 1.1 & 0.7 & 0.027 \\
\hline
\end{tabular}

Abbreviations: AN, women recovered from anorexia nervosa, restricting type; BDI, Beck Depression Inventory; CW, healthy control women; TCI, Temperament and Character Inventory; Y-BOCS, Yale-Brown Obsessive Compulsive Scale.

Group comparisons by Wilcoxon's rank-sum tests with exact significance levels.

$\mathrm{Df}=30$.

The significant values are bolded. 
Table 2 ANOVA Results Group $\times$ Time

\begin{tabular}{lll}
\hline Regions & $\mathbf{F}$ & $\mathbf{P}$ \\
\hline Insula left & $\mathbf{2 . 8 1 2}$ & $\mathbf{0 . 0 0 3}$ \\
Insula right & $\mathbf{2 . 2 0 9}$ & $\mathbf{0 . 0 2}$ \\
Orbital frontal cortex (BAII) left & 1.124 & 0.34 \\
Orbital frontal cortex (BAII) right & 2.298 & 0.23 \\
Amygdala left & 1.706 & 0.08 \\
Amygdala right & 1.395 & 0.19 \\
Anterior cingulate cortex & $\mathbf{1 . 8 7 7}$ & 0.05 \\
Anterior ventral striatum left & 1.335 & 0.22 \\
Anterior ventral striatum right & 1.147 & 0.33 \\
Dorsal caudate left & $\mathbf{2 . 2 1 2}$ & $\mathbf{0 . 0 2}$ \\
Dorsal caudate right & $\mathbf{2 . 0 4 0}$ & $\mathbf{0 . 0 3}$ \\
Dorsal putamen left & $\mathbf{2 . 7 2 7}$ & $\mathbf{0 . 0 0 4}$ \\
Dorsal putamen right & $\mathbf{2 . 6 6 3}$ & $\mathbf{0 . 0 0 5}$ \\
Middle caudate left & 1.860 & 0.06 \\
Middle caudate right & $\mathbf{2 . 2 6 2}$ & $\mathbf{0 . 0 2}$ \\
Ventral putamen left & $\mathbf{2 . 9 2 1}$ & $\mathbf{0 . 0 0 2}$ \\
Ventral putamen right & $\mathbf{2 . 3 2 1}$ & $\mathbf{0 . 0 1}$ \\
\hline Compaisons betwen & & \\
\hline
\end{tabular}

Comparisons between groups (women recovered from anorexia nervosa and controls) over time collapsed across conditions (sugar and water) using analyses of variance method are shown.

$\mathrm{Df}=1.30$.

The significant values are bolded.

their ratings of pleasantness and signal activity for sucrose for the right and left ventral putamen (left: $r=0.603$, $P=0.013$; right: $r=0.548, P=0.028$ ), dorsal putamen (left: $r=0.496, P=0.05$; right: $r=0.609, P=0.012)$, and anterior cingulate cortex $(r=0.564, P=0.023)$. In comparison, no relationships were found for the AN subjects between their ratings of pleasantness of the sucrose taste and any brain region. No relationship was found between perceived pleasantness of the taste of water and any brain region for either of the two groups.

We also asked subjects to rate their degree of anxious feelings during the taste procedure, as a means of assessing a dysphoric response to the taste stimuli. The signal activity of the insula when tasting sugar was not related to anxious feelings for CW if an outlier was removed (left: $r=-0.487$, $P=0.066$; right: $r=-0.334, P=0.224)$. Similarly, there was no relationship between anxiety and signal response to sugar in AN subjects in the insula (left: $r=-0.434$, $P=0.092$, right; $r=-0.187, P=0.487$ ) or any other ROI. In addition, there was also no relationship between anxiety and signal response to water in either group for all ROIs.

Mean percent signal changes in AN subjects for all ROIs were not related to age, BMI, length of recovery, or anxiety (STAI) (eg AN insula left: $r=0.249, P=0.351$; AN insula right: $r=-0.206, P=0.444$ ) or depression (BDI) (eg $\mathrm{AN}$ insula left: $r=0.053, P=0.846$; AN insula right: $r=-0.309$, $P=0.244)$.

\section{DISCUSSION}

This is the first study to use fMRI to investigate the effect of administration of nutrients to individuals with AN. This study provides evidence that individuals recovered from AN have disturbances of gustatory processing in the central nervous system. Compared to CW, the remitted AN subjects had a significantly reduced fMRI signal response to the blind administration of sucrose or water in the insula, anterior cingulate, and striatal regions. Importantly, AN and CW also showed differences in correlations between experience of pleasant taste and brain activation. For CW, self-ratings of pleasantness of the sugar taste were positively correlated to the signal response in the insula, anterior cingulate, and ventral and dorsal putamen. In comparison, AN individuals failed to show any relationship in these regions to self-ratings of pleasant response to a sucrose taste.

To our knowledge, only one previous study has used fMRI to investigate response to food in remitted $A N$ compared to healthy controls (Uher et al, 2003). However that study did not utilize actual taste stimuli, but showed pictures of food, a nongustatory stimulus, to subjects. Still, these investigators found that remitted AN subjects had altered activation of the anterior cingulate cortex and medial prefrontal regions, but did not report group differences in insula activation. While both of our studies show that remitted AN have an altered response to a food stimulus, the differences in methodology confounds a direct comparison of the findings.

Recovered AN individuals and CW showed differences in response to a taste of nutrients in the insula, as well as middle-dorsal caudate and putamen regions. Within each group, subjects had similar neural activation in response to sucrose and water. Others have found similar phenomena in healthy human subjects (Small et al, 2001; Rolls, 2005; De Araujo et al, 2003a). It is important to note that all five taste sensations activate the insula showing a chemotopical organization, but with a considerable overlap (Schoenfeld et al, 2004). In this study, we carried out an ROI analysis. By selecting a relatively large ROI for the insula, we may not have been able to distinguish the specific and perhaps different areas that are activated for sugar and water. That should be addressed in future studies.

Imaging methods used in this study were based on anatomical ROIs as a first pass at understanding the function of these regions. Discrimination of subregions of the insula is difficult because of the absence of landmarks. Functional divisions of the insula are based on cytoarchitectural, connectional, and behavioral studies in higher primates (Kaada et al, 1953; Mesulam and Mufson, 1982; Carmichael et al, 1994). In general, the anterior, less differentiated half of the insula receives the majority of projections from the amygdala and thalamic taste centers, making it an ideal site for the formulation of hedonic representations of taste. Consistent with this, anterior portions of the insula have been shown to be differentially activated to the intensity and valence of pleasant and unpleasant tastes (Small et al, 2003a), and are thus important to examine in the eating disordered population.

A large literature shows that the anterior insula and associated gustatory cortex responds not only to the taste and physical properties of food, but also to its rewarding properties (Schultz et al, 2000; O’Doherty et al, 2001; Small et al, 2001). Animal models show that fine tuning of feeding 

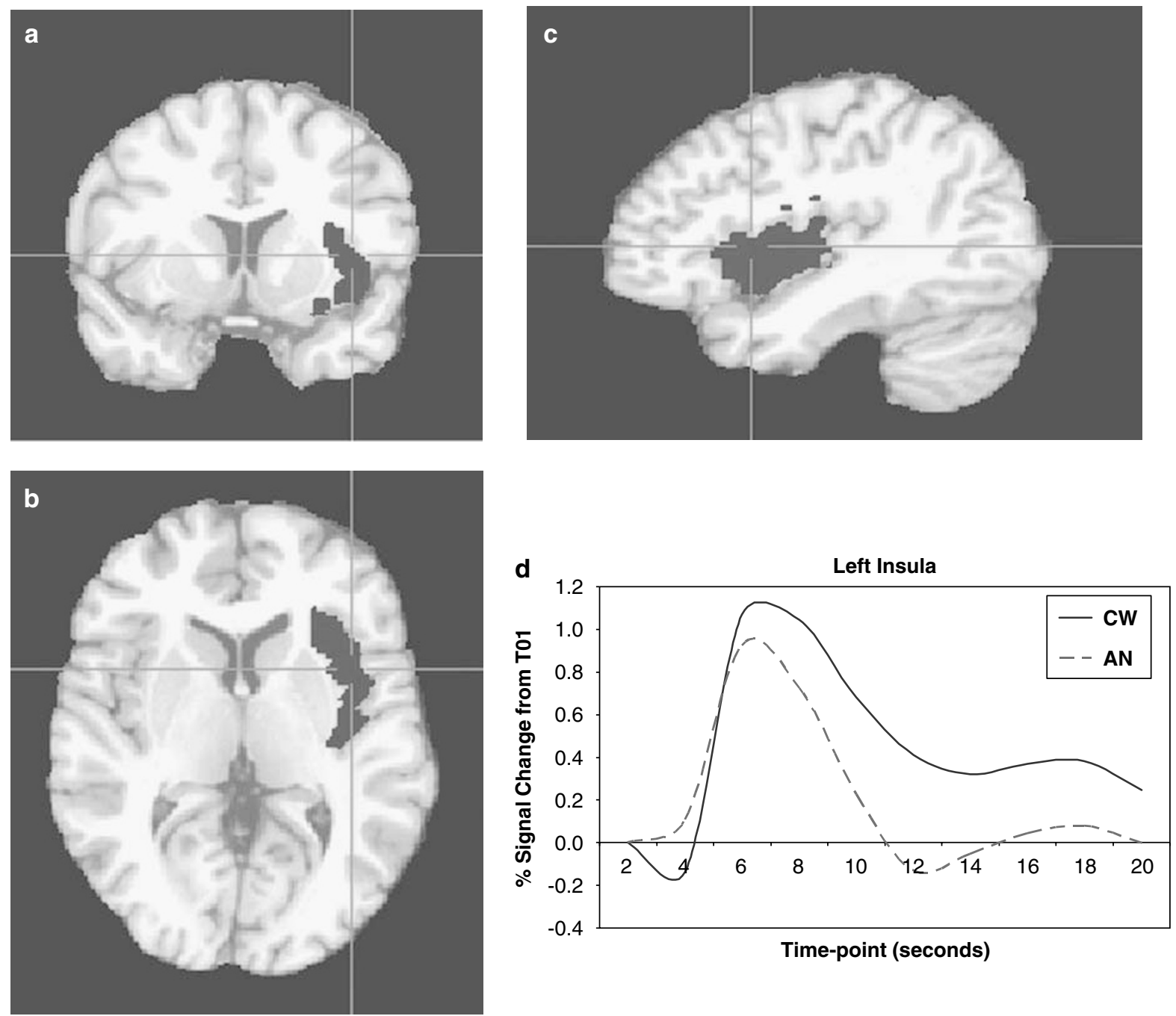

Figure I (a) Coronal, (b) axial, and (c) sagittal view of left insula ROI ( $x=-41, y=5, z=5$ ). (d) Time course of BOLD signal as a mean of all I6 recovered restricting-type anorexia nervosa and 16 control women for taste-related (sucrose and water) response in the left insula.

responses to salient food items are lost after insula lesions. For example, the natural devaluation of food after feeding to satiety is attenuated in lesioned animals, suggesting that the insula encodes representations of the incentive value of taste under specific conditions (Balleine and Dickinson, 2000). Similarly, appropriate avoidance of foods previously associated to sickening agents is lost after insula lesions, providing further evidence that the insula encodes the incentive value of taste in particular circumstances (Dunn and Everitt, 1988).

Our results in the insula are reinforced by parallel findings in basal ganglia subregions that receive insula inputs. While the entire striatum receives inputs from the insula as a whole, the ventral putamen is a specific recipient of inputs from anterior insular regions encompassing the gustatory cortex (Fudge et al, 2005). The ventral putamen in turn projects to the globus pallidus, another region with significant alterations in signal. The pattern of relatively decreased signal in these interconnected structures suggests a circuit-wide disturbance in recovered AN. Insular inputs to the striatum are hypothesized to mediate behaviors involving eating, particularly of highly palatable, high energy foods. The ventrolateral striatal subregions, including the ventral putamen, have been especially implicated (Kelley et al, 2002). AN subjects tend to avoid high calorie, palatable food. In theory, this is consistent with abnormal responses of insula-striatal circuits that are hypothesized to mediate behavioral responses to the incentive value of food.

The insula, and these connected regions, are thought to play an important role in processing interoceptive information, which can be defined as the sense of the physiological condition of the entire body (Craig, 2002). Aside from taste, interoceptive information includes sensations such as temperature, touch, muscular and visceral sensations, vasomotor flush, air hunger, and others (Paulus and Stein, 2006). The role of the insula is thus focused on how the value of stimuli might affect the body state. The involvement of the circuit that processes introceptive stimuli in $\mathrm{AN}$ raises the question of whether these individuals have a specific disturbance of taste modulation, or a more generalized disturbance related to the integration of interoceptive stimuli. The possibility that this is a more generalized phenomenon is supported by another fMRI 

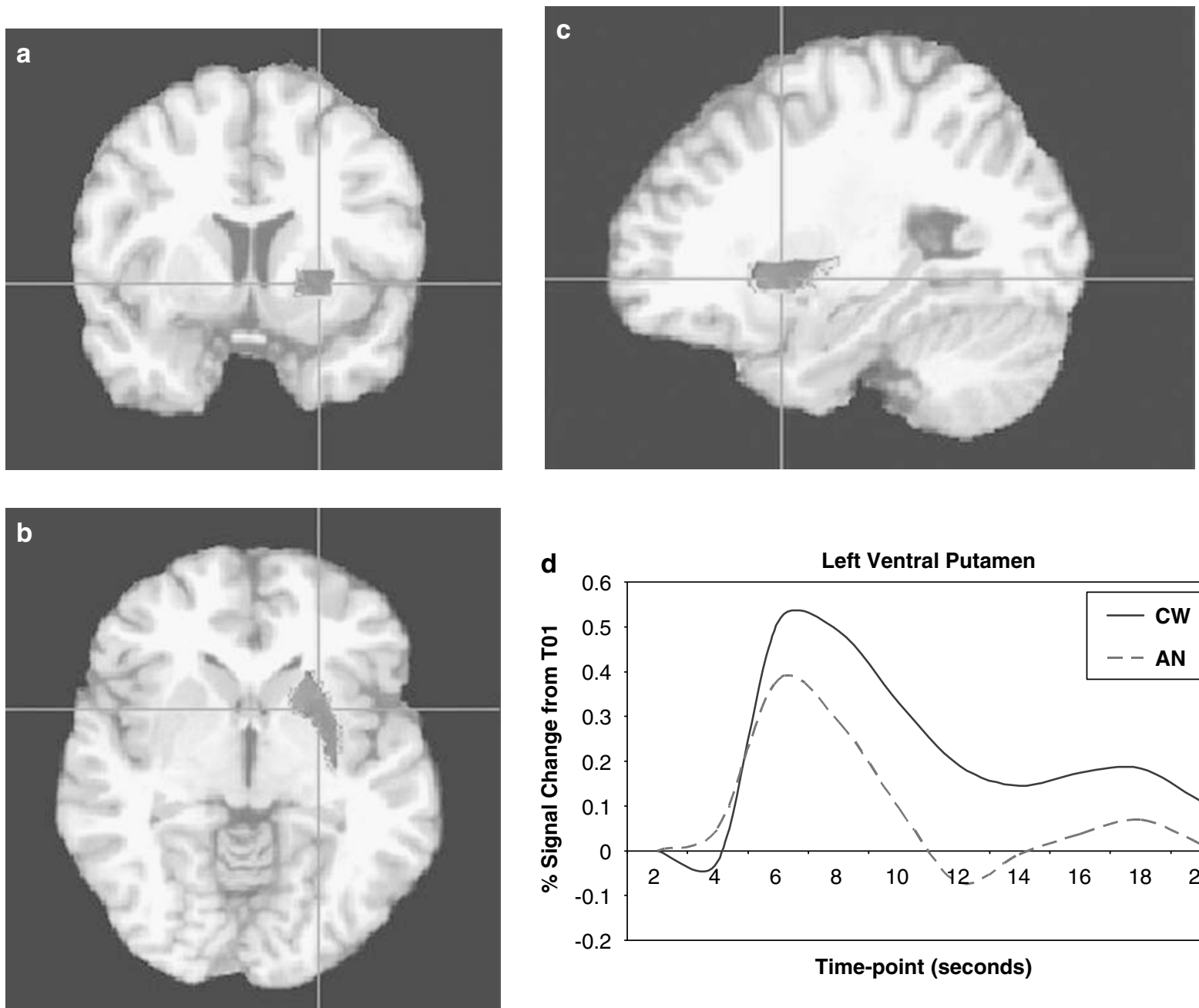

d

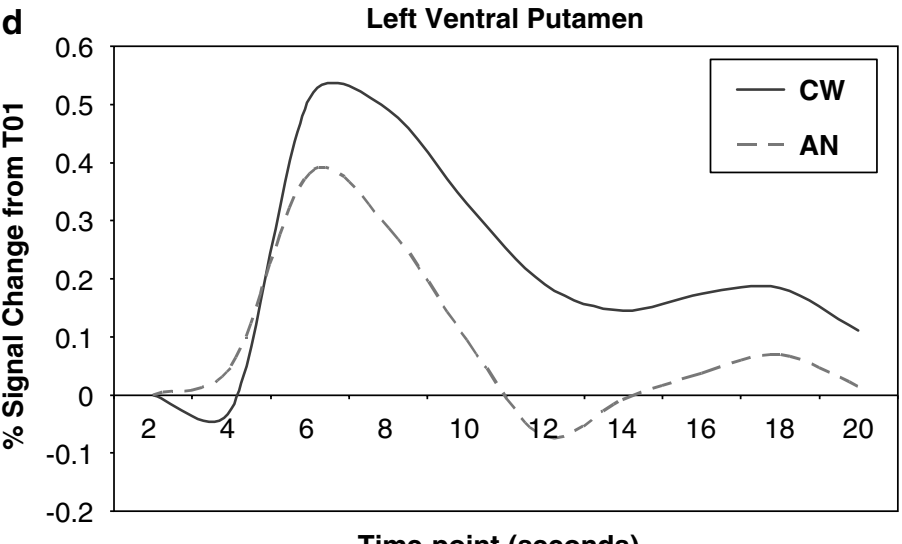

Time-point (seconds)

Figure 2 (a) Coronal, (b) axial, and (c) sagittal view of the left ventral putamen $\mathrm{ROI}(x=-20, y=8, z=0)$. (d) Time course of BOLD signal as a mean of all 16 women recovered from restricting-type anorexia nervosa and 16 control women for taste-related (sucrose and water) response in the left ventral putamen.

study from our group that used a monetary reward task (Wagner et al, 2005). That study found that recovered AN subjects had altered discrimination of positive and negative stimuli in ventral and dorsal striatal regions. As noted by Paulus and Stein (2006), interoception has long been thought to be critical for self-awareness because it provides the link between cognitive and affective processes and the current body state. Many of the symptoms commonly found in $\mathrm{AN}$, such as distorted body image, lack of recognition of the symptoms of malnutrition, such as a failure to appropriately respond to hunger, and diminished motivation to change, could be related to disturbed introceptive awareness.

Importantly, only controls, but not recovered AN, showed a positive relationship between self-ratings of pleasantness and the intensity of the signal for sugar in the insula, ventral, and dorsal putamen as well as anterior cingulate cortex. Consistent with these findings Small et al (2001, 2003a) found valence-specific responses in the anterior insula/operculum, OFC, and anterior cingulate cortex in healthy subjects. In contrast, amygdala activity was driven by stimulus intensity irrespective of valence. We used only one stimulus intensity (water or $10 \%$ sucrose), and did not find responses in the amygdala, possibly supporting the idea that the amygdala is less involved in coding the valence (pleasantness) of the stimulus.

When we designed this study, we had concerns that an anxious response to the taste of a sugar might contribute to an altered response in AN. That is because individuals prone to anxiety often have heightened activity in the anterior insula during processing of certain kinds of salient stimuli (Paulus and Stein, 2006). We found no difference in response to sucrose and water in either group. However, there was a trend toward a negative relationship with selfratings of anxiety in the left insula when tasting sugar in the AN subjects and a positive relationships with self-ratings of pleasure in CW. Potential contributions of emotional valance should be explored in future studies.

Some studies claim that the OFC is responsive to hedonic properties and the insula is not (De Araujo et al, 2003b; Kringelbach et al, 2003; Rolls, 2005). We found no difference between groups in terms of activation to sugar or water and no correlations between pleasantness ratings and neural activity in the portion of OFC considered 
a

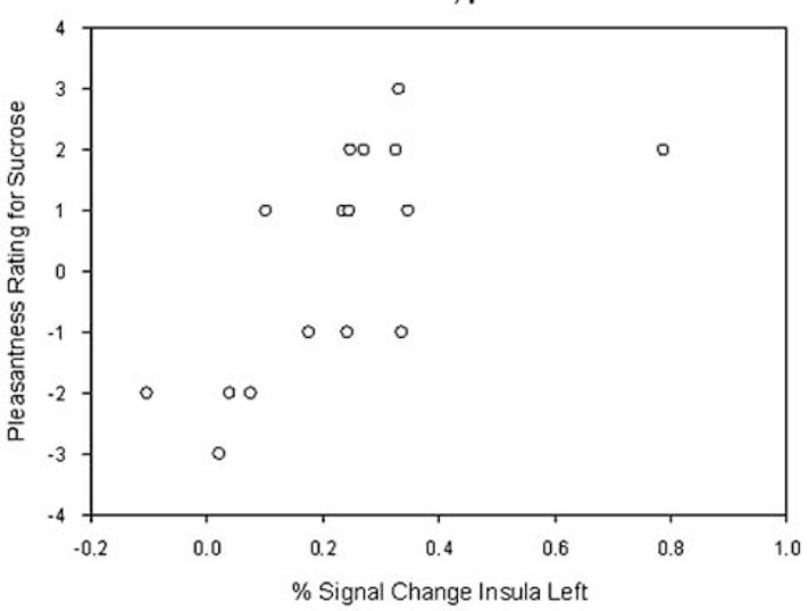

C

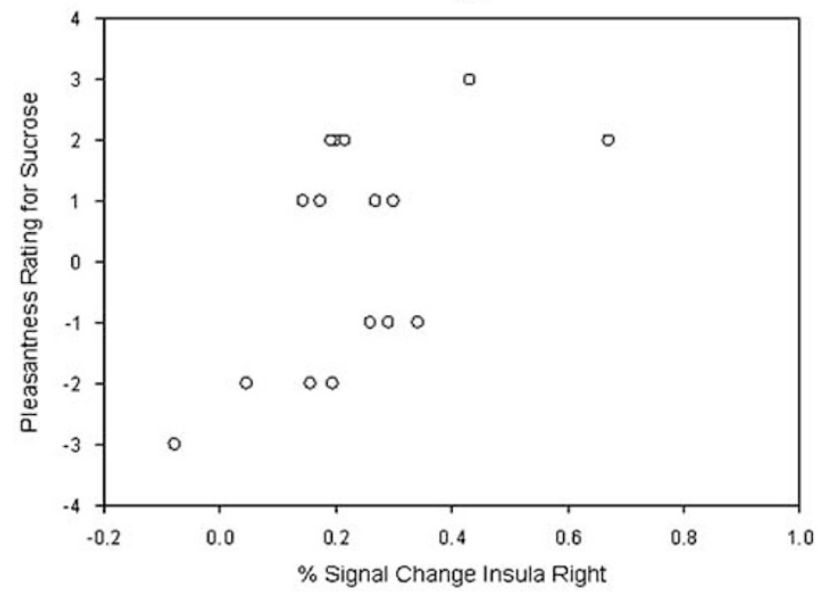

b

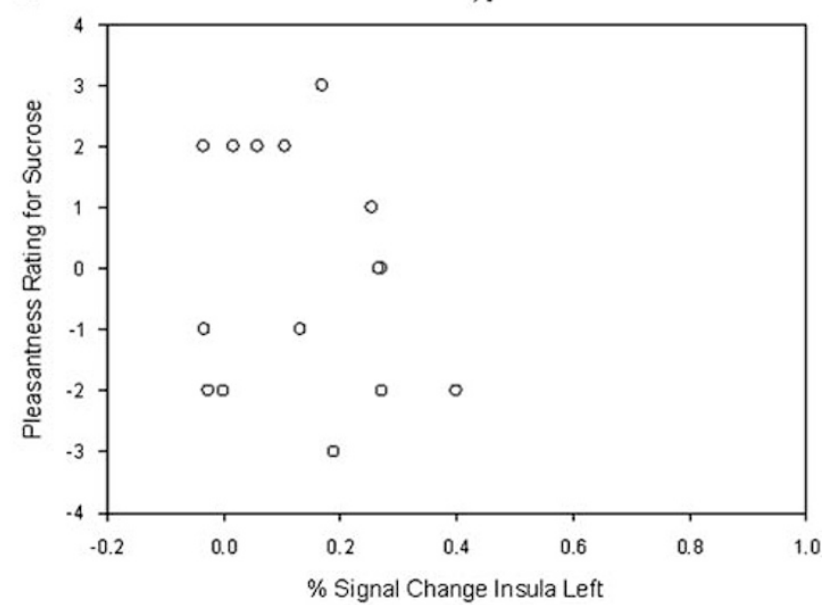

d

AN: $r=0.201, p=0.473$

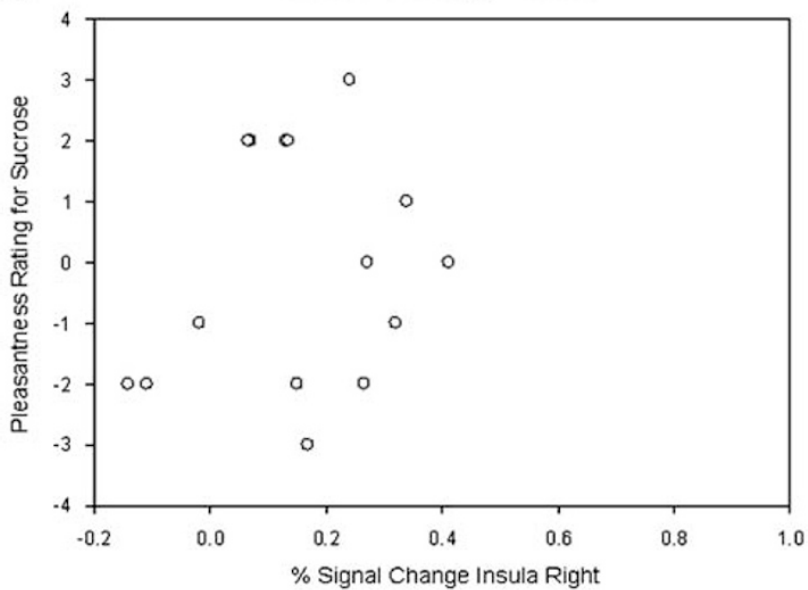

Figure 3 Correlation between pleasantness ratings for sucrose and mean percentage signal change in the left insula for (a) CW or (b) AN and the right insula for (c) CW or (d) AN. CW, control women; AN, women recovered from restricting-type anorexia nervosa.

'secondary gustatory cortex'. We did not examine the entire OFC, rather focused on BA11 described previously in gustatory-related studies (O'Doherty et al, 2000). Neurons in the secondary gustatory cortex may be preferentially tuned to satiety state (Rolls et al, 1981; Rolls, 2005). Since subjects in this study were fed to satiety before testing, the absence of activation of the OFC could be due to sensoryspecific satiety effects. It is important to note that the anterior insula occupies the caudal aspect of the OFC in higher primates. The distinction between the ventral OFC (including the secondary gustatory cortex) and anterior insula cannot therefore be reliably distinguished in functional imaging studies. In addition, the expectation of a sweet taste, in contrast to a neutral taste, results in activation of OFC (and amygdala and anterior striatum), while the receipt of both stimuli activates only a very lateral area of the OFC that looks like anterior insula (O'Doherty et al, 2002). Therefore the lack of anticipation of stimuli in our design could account for the lack of OFC (BA11) activation, as well as lack of amygdala and anterior ventral striatal activation in our study.

The lack of AVS and OFC findings could also be due to susceptibility artifact from the frontal sinus, which decreases the signal-to-noise ratio, and therefore further lowers the power to detect differences in these regions. Although our study was designed to minimize susceptibility artifact by using a reverse spiral acquisition (Stenger et al, 1999), susceptibility artifact in the AVS and OFC remains a concern.

\section{Limitations/Future Directions}

A considerable literature has focused on determining how feeding-related circuits work in healthy people. This is the first study of this type to examine some of these functional pathways in $\mathrm{AN}$, a complex disease with eating disturbance as a core feature. To more precisely understand brain circuitry underlying eating behavior in $\mathrm{AN}$, future studies employing voxel-based measures will be important. Furthermore, assessing which taste stimuli, and their optimal intensity, are 'pleasant' for AN requires more study. For example, we decided to use a $10 \%$ sucrose because this percent is assumed to be pleasant for many people (Drewnowski et al, 1987; Sunday and Halmi, 1990). But it is possible that other intensities of sugar might show different results. 
Many factors may influence these findings, including anticipatory anxiety, mood status, laterality or pre-study food ingestion. Future studies should address these issues, as well as the response to other foods, such as dietary fat.

Functional MRI group analyses are potentially confounded by differences in neuroanatomy between the groups, which can lead to inconsistent alignment of the functional MRI data into a common anatomic space. A recent study from our group, including some of these subjects, showed normal brain volume after recovery from AN (Wagner et al, 2006b). Still, it is possible that the recovered AN brains may systematically have more alignment error than the control subjects. In the current study we addressed this by using a single-subject ROI-based analysis, focusing on regions for which we had initial hypotheses. Our approach limits errors due to poor registration by selecting the ROI separately for each subject, using a non-linear warp and segmentation, to define the anatomic ROIs for each subject (Wu et al, 2006). This ROIbased approach, however, is limited to a priori pre-defined ROIs. Thus, for instance, we are unable to identify whether specific sub-regions of the insula are differentially activated by taste in these groups. However, discrimination of subregions of the insula is difficult because of the absence of landmarks. Functional divisions of the insula are based on cytoarchitectural, connectional and behavioral studies in higher primates (Kaada et al, 1953; Mesulam and Mufson, 1982; Carmichael et al, 1994). In general, the anterior, less differentiated half of the insula receives the majority of projections from the amygdala and thalamic taste centers, making it an ideal site for the formulation of hedonic representations of taste. Consistent with this, anterior portions of the insula have been shown to be differentially activated to the intensity and valence of pleasant and unpleasant tastes (Small et al, 2003a,b), and are thus important to examine in the eating disordered population in future studies.

Finally, AN is a psychiatric disorder with disturbances in mood and anxiety regulation in addition to core eating dysregulation. Processing of emotionally relevant stimuli occurs in prefrontal cortex, OFC, and amygdala/striatal regions, and has been found to be aberrant in patients with major depression and anxiety disorder (Keedwell et al, 2005; Tremblay et al, 2005; Forbes et al, 2007; Pillay et al, 2006). Comparing AN individuals to psychiatric controls with mood and anxiety findings, as well as normal controls, will eventually be important in determining whether aberrant feeding behaviors are related to more general anxiety and mood phenomena. The cross-sectional design of this study does not permit determining whether these findings are a trait that contributes to the onset of AN, or a 'scar' that is the consequence of past malnutrition and weight loss.

In summary, this is the first study to show that a dysregulation of the insula and related regions in response to taste of nutrients occurs in AN. This supports the possibility that individuals with $\mathrm{AN}$ do, in fact, have disturbances of appetite regulation. The wealth of studies in healthy humans and non-human primates provides a body of knowledge that can be used to begin to understand the pathophysiology of this devastating and difficult to treat illness.

\section{ACKNOWLEDGEMENTS}

We thank Eva Gerardi for the manuscript preparation. The authors are indebted to the participating subjects for their contribution of time and effort in support of this study.

\section{DISCLOSURE/CONFLICT OF INTEREST}

Funding for this work was provided by the National Institute of Mental Health (NIMH) MH046001, MH04298, and K05-MH01894 and grant support from Erwin-Schrödinger Research Fellowship of the Austrian Science Fund (J2188 and J2359-B02) to Ursula Bailer. Dr Kaye has received salary support from the University of Pittsburgh and the University of California, San Diego; Research funding/support from the NIMH; Research funding for an investigator initiated treatment study from Astra-Zeneca and consulting fees from Lundbeck. In addition, there are honoraria for presentations from academic institutions and meetings, and compensation for grant review activities from the National Institutes of Health.

The remaining authors, A Wagner, $\mathrm{H}$ Aizenstein, L Mazurkewicz, J Fudge, G Frank, K Putnam, and L Fischer declare that, except for income received from our primary employers and the above-mentioned funding, no further financial support or compensation has been received from any individual or corporate entity over the past 3 years for research or professional service and there are no personal financial holdings that could be perceived as constituting a potential conflict of interest.

\section{REFERENCES}

American Psychiatric Association (1994). Diagnostic and Statistical Manual of Mental Disorders, 4th edn. American Psychiatric Association: Washington, DC.

APA (2000). Diagnostic and Statistical Manual of Mental Disorders, Fourth edition, Text Revision (DSM-IV-TR). American Psychiatric Association: Washington, DC.

Bailer UF, Kaye WH (2003). A review of neuropeptide and neuroendocrine dysregulation in anorexia and bulimia nervosa. Curr Drug Targets: CNS Neurol Disord 2: 53-59.

Balleine B, Dickinson A (2000). The effect of lesions of the insular cortex on instrumental conditioning evidence for a role in incentive memory. J Neurosci Methods 20: 8954-8964.

Beck AT, Ward M, Mendelson M, Mock J, Erbaugh J (1961). An inventory for measuring depression. Arch Gen Psychiatry 4: 53-63.

Bornert P, Aldefeld B, Eggers H (2000). Reversed spiral MR imaging. Magn Reson Med 44: 479-484.

Bulik C, Sullivan PF, Tozzi F, Furberg H, Lichtenstein P, Pedersen NL (2006). Prevalence, heritability and prospective risk factors for anorexia nervosa. Arch Gen Psychiatry 63: 305-312.

Carmichael ST, Clugnet MC, Price JL (1994). Central olfactory connections in the macaque monkey. J Comp Neurol 346: 403-434.

Cloninger CR, Przybeck TR, Svrakic DM, Wetzel RD (1994). The Temperament and Character Inventory (TCI): A Guide to its Development and Use. Center for Psychobiology of Personality, Washington University: St Louis, MO.

Couturier J, Lock J (2006). What is recovery in adolescent anorexia nervosa? Int J Eat Disord 39: 550-555.

Craig AD (2002). How do you feel? Interoception: the sense of the physiological condition of the body. Nat Rev Neurosci 3: 655-666. 
De Araujo I, Kringelbach M, Rolls ET, McGlone F (2003a). Human cortical responses to water in the mouth, and the effects of thirst. J Neurophysiol 90: 1865-1876.

De Araujo I, Rolls E, Kringelbach M, McGlone F, Phillips N (2003b). Taste-olfactory convergence, and the representation of the pleasantness of flavour, in the human brain. Eur J Neurosci 18: 2059-2068.

Drevets WC, Gautier C, Price JC, Kupfer DJ, Kinahan PE, Grace AA et al (2001). Amphetamine-induced dopamine release in human ventral striatum correlates with euphoria. Biol Psychiatry 49: 81-96.

Drewnowski A, Halmi KA, Pierce B, Gibbs J, Smith GP (1987). Taste and eating disorders. Am J Clin Nutr 46: 442-450.

Dunn L, Everitt B (1988). Double dissociations of the effects of amygdala and insular cortex lesions on conditioned taste aversion, passive avoidance, and neophobia in the rat using excitotoxin ibotenic acid. Behav Neurosci 102: 3-23.

Ellison Z, Foong J, Howard R, Bullmore E, Williams S, Treasure J (1998). Functional anatomy of calorie fear in anorexia nervosa. Lancet 352: 1192.

Faurion A, Cerf B, Van De Moortele PF, Lobel E, Mac Leod P, Le Bihan D (1999). Human taste cortical areas studied with functional magnetic resonance imaging: evidence of functional lateralization related to handedness. Neurosci Lett 277: 189-192.

Forbes EE, Shaw DS, Dahl RE (2007). Alterations in reward-related decicion making in boys with recent and future depression. Biol Psychiatry 61: 633-639.

Frank G, Bailer UF, Henry S, Drevets W, Meltzer CC, Price JC et al (2005). Increased dopamine D2/D3 receptor binding after recovery from anorexia nervosa measured by positron emission tomography and $\left[{ }^{11} \mathrm{C}\right]$ raclopride. Biol Psychiatry 58: 908-912.

Frank G, Kaye W, Carter C, Brooks S, May C, Fissel K et al (2003). The evaluation of brain activity in response to taste stimuli-a pilot study and method for central taste activation as assessed by event related fMRI. J Neurosci Methods 131: 99-105.

Fudge J, Breitbart M, Danish M, Pannoni V (2005). Insular and gustatory inputs to the caudal ventral striatum in primates. J Comp Neurol 490: 101-118.

Gordon CM, Dougherty DD, Fischman AJ, Emans SJ, Grace E, Lamm $\mathrm{R}$ et al (2001). Neural substrates of anorexia nervosa: a behavioral challenge study with positron emission tomography. J Pediatr 139: 51-57.

Herzog DB, Nussbaum KM, Marmor AK (1996). Comorbidity and outcome in eating disorders. Psychiatr Clin North Am 19: 843-859.

Kaada BR, Jansen J, Andersen P (1953). Stimulation of the hippocampus and medial cortical areas in unanesthetized cats. Neurology 3: 844-857.

Keedwell PA, Andrew C, Williams SC, Brammer MJ, Phillips ML (2005). The neural correlates of anhedonia in major depressive disorder. Biol Psychiatry 58: 843-853.

Kelley AE, Bakshi VP, Haber SN, Steininger T, Will MJ, Zhang M (2002). Opioid modulation of taste hedonics within ventral striatum. Physiol Behav 76: 365-377.

Kringelbach ML, O’Doherty J, Rolls E, Andrews C (2003). Activation of the human orbitofrontal cortex to a liquid food stimulus is correlated with its subjective pleasantness. Cereb Cortex 13: 1064-1071.

McKenzie JM, Joyce PR (1992). Hospitalization for anorexia nervosa. Int J Eat Disord 11: 235-241.

Mesulam MM, Mufson EJ (1982). Insula of the old world mokey. I. Architectonics in the insulo-orbito-temporal component of the paralimbic brain. J Comp Neurol 212: 1-22.

Metropolitan Life Insurance Company (1959). New Weight Standards for Men and Women. Stat Bull Metrop Insur Co 40: $1-11$.
Mirenowicz J, Schultz W (1994). Importance of unpredictability for reward responses in primate dopamine neurons. J Neurophysiol 72: $1024-1027$.

Naruo T, Nakabeppu Y, Sagiyama K, Munemoto T, Homan N, Deguchi D et al (2000). Characteristic regional cerebral blood flow patterns in anorexia nervosa patients with binge/purge behavior. Am J Psychiatry 157: 1520-1522.

Noll DC, Cohen JD, Meyer CH, Schneider W (1995). Spiral K-space MR imaging of cortical activation. J Magn Res Imaging 5: 49-56.

Nozoe S, Naruo T, Nakabeppu Y, Soejima Y, Nakajo M, Tanaka H (1993). Changes in regional cerebral blood flow in patients with anorexia nervosa detected through single photon emission tomography imaging. Biol Psychiatry 34: 578-580.

O’Doherty J, Kringelbach ML, Rolls ET, Hornak J, Andrews C (2001). Abstract reward and punishment representations in the human orbitofrontal cortex. Nat Neurosci 4: 95-102.

O’Doherty J, Rolls ET, Francis S, Bowtell R, McGlone F, Kobal G et al (2000). Sensory-specific satiety-related olfactory activation of the human orbitofrontal cortex. Neuroreport 11: 893-897.

O’Doherty JP, Deichmann R, Critchley HD, Dolan RJ (2002). Neural responses during anticipation of a primary taste reward. Neuron 33: 815-826.

Paulus M, Stein MB (2006). An insular view of anxiety. Biol Psychiatry 60: 383-387.

Pillay S, Gruber S, Rogowska J, Simpson N, Yurgelun-Todd D (2006). fMRI of fearful facial affect recognition in panic disorder: the cingulate gyrus-amygdala connection. J Affect Disord 94: 173-181.

Rolls B, Rolls E, Rowe E, Sweeney K (1981). Sensory specific satiety in man. Physiol Behav 27: 137-142.

Rolls ET (2005). Taste, olfactory, and food texture processing in the brain, and the control of food intake. Physiol Behav 85: 45-56.

Schoenfeld M, Neuer G, Tempelmann C, Schussler K, Noesselt T, Hopf J et al (2004). Functional magnetic resonance tomography correlates of taste perception in the human primary taste cortex. Neurosci 127: 347-353.

Schultz W, Tremblay L, Hollerman JR (2000). Reward processing in primate orbitofrontal cortex and basal ganglia. Cereb Cortex 10: $272-284$.

Schultz W (1998). Predictive reward signal of dopamine neurons. J Neurophysiol 80: 1-27.

Scott TR, Yaxley S, Sienkiewicz Z, Rolls E (1986). Gustatory responses in the frontal opercular cortex of the alert cynomolgus monkey. J Neurophysiol 56: 876-890.

Small D, Gregory M, Mak Y, Gitelman D, Mesulam M, Parrish T (2003a). Dissociation of neural representation of intensity and affective valuation in human gustation. Neuron 39: 701-711.

Small D, Jones-Gotman M, Dagher A (2003b). Feeding-induced dopamine release in dorsal striatum correlates with meal pleasantness ratings in healthy human volunteers. Neuroimage 19: $1709-1715$.

Small D, Zatorre R, Dagher A, Evans A, Jones-Gotman M (2001). Changes in brain activity related to eating chocolate: from pleasure to aversion. Brain 124: 1720-1733.

Spielberger CD, Gorsuch RL, Lushene RE (1970). STAI Manual for the State Trait Anxiety Inventory. Consulting Psychologists Press: Palo Alto, CA.

Stein DJ, Arya M, Pietrini P, Rapoport JL, Swedo SE (2006). Neurocircuitry of disgust and anxiety in obsessive compulsive disorder: a positron emission tomography study. Metab Brain Dis 21: 267-277.

Stenger VA, Peltier S, Boada FE, Noll DC (1999). 3D spiral cardiac/ respiratory ordered fMRI data acquisition at 3 Tesla. Magn Reson Med 41: 983-991.

Sullivan PF (1995). Mortality in anorexia nervosa. Am J Psychiatry 152: $1073-1074$. 
Sunday SR, Halmi KA (1990). Taste perceptions and hedonics in eating disorders. Physiol Behav 48: 587-594.

Tremblay LK, Naranjo CA, Graham SJ, Herrmann N, Mayberg HS, Hevenor SJ et al (2005). Functional neuroanatomical substrates of altered reward processing in major depressive diorder revealed by a dopaminergic probe. Arch Gen Psychiatry 62: $1228-1236$.

Tzourio-Mazoyer N, Landeau B, Papathanassiou D, Crivello F, Etard O, Delcroix N et al (2002). Automated anatomical labeling of activations in SPM using a macroscopic anatomical parcellation of the MNI MRI single-subject brain. Neuroimage 15: 273-289.

Uher R, Brammer M, Murphy T, Campbell I, Ng V, Williams S et al (2003). Recovery and chronicity in anorexia nervosa: brain activity associated with differential outcomes. Biol Psychiatry 54: 934-942.

Uher R, Murphy T, Brammer M, Dalgleish T, Phillips M, Ng V et al (2004). Medial prefrontal cortex activity associated with symptom provocation in eating disorders. Am J Psychiatry 161: 1238-1246.

Wagner A, Barbarich-Mastseller N, Frank G, Bailer UF, Weissfeld L, Wonderlich S et al (2006a). Personality traits after recovery from eating disorders: do subtypes differ? Int J Eat Disord 39: 276-284.
Wagner A, Greer P, Bailer U, Frank G, Henry S, Putnam K et al (2006b). Normal brain tissue volumes after long-term recovery in anorexia and bulimia nervosa. Biol Psychiatry 59: 291-293.

Wagner A, May C, Alzenstein H, Frank GK, Figurski J, Bailer UF et al (2005). Reward-related neural responses in anorexia and bulimia nervosa after recovery using functional magnetic resonance imaging. Biol Psychiatry 57: 8S, 709.

Woods RP, Grafton ST, Holmes CJ, Cherry SR, Mazziotta JC (1998). Automated image registration: I. General methods and intrasubject, intramodality validation. J Comput Assist Tomogr 22: $139-152$.

Wu M, Carmichael O, Lopez-Garcia P, Carter CS, Aizenstein H (2006). Quantitative comparison of AIR, SPM and the fully deformable model for atlas-based segmentation of functional and structural MR images. Hum Brain Mapp 27: 747-754.

Yaxley S, Rolls E, Sienkiewicz Z (1990). Gustatory responses of single neurons in the insula of the macaque monkey. J Neurophysiol 63: 689-700.

Zald D, Pardo J (2000). Cortical activation induced by intraoral stimulation with water in humans. Chem Senses 25: 267-275.

Zhang H, D'Souza D, Raap DK, Francisca G, Battaglia G, Muma NA et al (2001). Characterization of the functional heterologous densitization of hypothalamic $5-\mathrm{HT}_{1 \mathrm{~A}}$ receptors after $5-\mathrm{HT}_{2 \mathrm{~A}}$ receptor activation. J Neurosci 21: 7919-7927. 